\title{
Operator Regularization and
}

\section{Noncommutative Chern Simons Theory}

\author{
D.G.C. McKeon \\ Department of Applied Mathematics \\ University of Western Ontario \\ London \\ CANADA
}

N6A 5B7

Tel: 519-661-2111, ext. 88789

Fax: 519-661-3523

Email: DGMCKEO2@UWO.CA

\section{Abstract}

We examine noncommutative Chern Simons theory using operator regularization. Both the $\zeta$-function and the $\eta$-function are needed to determine one loop effects. The contributions to these functions coming from the two point function is evaluated. The $U(N)$ noncommutative model smoothly reduces to the $S U(N)$ commutative model as the noncommutative parameter $\theta_{\mu \nu}$ vanishes. 


\section{Introduction}

Normally, Chern Simons theory is a purely topological theory as it is metric independent [1-3]. However, if we consider Chern Simons theory in a noncommutative space [4,5] in which

$$
\left[x_{\mu}, x_{\nu}\right]=-i \theta_{\mu \nu}
$$

metric dependence inevitably arise. The consequences of the presence of $\theta_{\mu \nu}$ in noncommutative Chern Simons theory have been considered in [6]. In particular, the one loop contribution to the two point function has been computed in [7].

In this paper, we would like to examine this one loop, two point function using operator regularization [8], a generalization of $\zeta$-function regularization [9]. The modulus and phase of the functional determinant associated with the effective action at one loop order are associated with the $\zeta$-function $[8,9]$ and $\eta$-function $[1,10-12]$ respectively. This approach has been used in conventional Chern Simons theory [11-15].

\section{The $\zeta$ - and $\eta$-functions}

The usual Chern Simons action is given by

$$
S=\int d^{3} x \operatorname{Tr} \epsilon_{i j k}\left(A_{i} \partial_{j} A_{k}-\frac{2 i}{3} A_{i} A_{j} A_{k}\right)
$$

where $A_{i}=A_{i}^{a} T^{a}$ with $T^{a}$ being the generator of a Lie group $G$. If $G$ is a unitary group $U(N)$, then (2) can be generalized by converting the products occuring in (2) to Moyal 
product in which $[4,5]$

$$
A_{i}(x) * A_{j}(x)=\left.e^{-\frac{i}{2} \partial^{x} \times \partial^{y}} A_{i}(x) A_{j}(y)\right|_{x=y}
$$

where $a \times b=\theta_{i j} a_{i} b_{j}$.

In order to use operator regularization to compute radiative effects, it is necessary to employ background field quantization [16-18]. We begin by splitting $A_{i}$ into the sum of background and quantum fields

$$
A_{i} \rightarrow A_{i}+Q_{i}
$$

A gauge fixing Lagrangian

$$
L_{g f}=\operatorname{Tr}\left(N D_{i}(A) Q_{i}\right)
$$

is chosen so as to leave the symmetry

$$
\begin{gathered}
A_{i} \rightarrow A_{i}+D_{i}(A) \Omega \\
Q_{i} \rightarrow Q_{i}-i\left(Q_{i} * \Omega-\Omega * Q_{i}\right) \\
\equiv Q_{i}-i\left[Q_{i}, \Omega\right]
\end{gathered}
$$

present in (2) unbroken. In (5), $N$ is a Nakanishi-Lautraup field and $D_{i}(A)$ is the covariant derivative

$$
D_{i}(A) f=\partial_{i} f-i\left[A_{i}, f\right] .
$$

The ghost Lagrangian associated with the gauge fixing of eq. (5) and the gauge transformation

$$
A_{i} \rightarrow A_{i}+D_{i}(A+Q) \Lambda
$$




$$
Q_{i} \rightarrow Q_{i}
$$

is

$$
L_{\text {ghost }}=\bar{C} D_{i}(A) D_{i}(A+Q) C .
$$

From (2), (5) and (9) it is evident that the terms in the effective Lagrangian $L+L_{g f}+L_{\text {ghost }}$ that are bilinear in the quantum fields are

$$
\begin{gathered}
L^{(2)}=\left(Q_{i}^{a}, N^{a}\right)\left(\begin{array}{cc}
\epsilon_{i p j} D_{p}^{a b}(A) & -D_{i}^{a b}(A) \\
D_{j}^{a b}(A) & 0
\end{array}\right)\left(\begin{array}{c}
Q_{j}^{b} \\
N^{b}
\end{array}\right) \\
+\bar{C}^{a}\left(D_{i}^{a b}(A) D_{i}^{b c}(A)\right) C^{c},
\end{gathered}
$$

so that the one loop generating functional is given by

$$
W^{(1)}(A)=\left(\ln \operatorname{det}^{-1 / 2} H_{I}\right)+\left(\ln \operatorname{det} H_{I I}\right)
$$

where $H_{I}$ and $H_{I I}$ are the two operators in (10). As $H_{I}$ is linear in derivatives, it is necessary to make the replacement

$$
\ln \operatorname{det}^{-1 / 2} H_{I} \rightarrow \ln \operatorname{det}^{-1 / 4} H_{I}^{2} .
$$

(A possible loss of phase in this replacement will be considered below.) Since

$$
\epsilon_{i j k} D_{j}(A) D_{k}(A) f=-\frac{i}{2} \epsilon_{i j k}\left[F_{j k}, f\right]
$$

where

$$
F_{j k}=\partial_{i} A_{j}-\partial_{j} A_{i}-i\left(A_{i} * A_{j}-A_{j} * A_{i}\right),
$$

it is evident that

$$
H_{I}^{2}=\left(\begin{array}{cc}
-D^{2} \delta_{i j} & -\epsilon_{i m n} F_{m n} \\
\epsilon_{m n j} F_{m n} & -D^{2}
\end{array}\right)
$$


We note that

$$
\begin{aligned}
& {[M, N]=} M^{a} T^{a} * N^{b} T^{b}-N^{b} T^{b} * M^{a} T^{a} \\
&= \frac{1}{2}\left(\left[T^{a}, T^{b}\right]\left\{M^{a}, N^{b}\right\}\right. \\
&\left.+\left\{T^{a}, T^{b}\right\}\left[M^{a}, N^{b}\right]\right) \\
& \equiv\left(i f^{a b c}\left\{M^{a}, N^{b}\right\}+d^{a b c}\left[M^{a}, N^{b}\right]\right) T^{c}
\end{aligned}
$$

and this becomes, on account of the Moyal product defined in (3)

$$
=2 i\left[f^{a b c} \cos \left(\frac{p \times q}{2}\right)+d^{a b c} \sin \left(\frac{p \times q}{2}\right)\right]\left(M^{a} N^{b} T^{c}\right)
$$

where $p$ and $q$ are the momenta of $M^{a}$ and $N^{b}$ respectively. This is to be utilized when employing a perturbative expansion of $W^{(1)}(A)$ in powers of $A$. (Conventions for $f^{a b c}$ and $d^{a b c}$ are those of [19].)

In operator regularization, one first uses

$$
\begin{aligned}
& \ln \operatorname{det} H=\operatorname{tr} \ln H \\
& =\lim _{s \rightarrow 0}-\frac{d}{d s}\left(t r H^{-s}\right) \\
& =-\left.\frac{d}{d s}\right|_{0} \frac{1}{\Gamma(s)} \int_{0}^{\infty} d t t^{s-1} t r e^{-H t} \\
& \equiv-\zeta^{\prime}(0)
\end{aligned}
$$

and then employs an expansion due to Schwinger [20]

$$
\begin{aligned}
& \operatorname{tr} e^{-\left(H_{0}+H_{1}\right) t}=\operatorname{tr}\left[e^{-H_{0} t}+\frac{(-t)}{1} e^{-H_{0} t} H_{1}\right. \\
& \left.+\frac{(-t)^{2}}{2} \int_{0}^{1} d u e^{-(1-u) H_{0} t} H_{1} e^{-u H_{0} t} H_{1}+\ldots\right]
\end{aligned}
$$


to effect an expansion in powers of the background field. (The dependence of $H$ on the background field resides entirely in $H_{1}$.)

It is now possible to apply (18) and (19) to compute the contribution of the two point function to the $\zeta$ function associated with $\ln \operatorname{det}^{-1 / 4} H_{I}^{2}$ and $\ln \operatorname{det} H_{I I}$. We find that

$$
W^{(1)}(A)=-\left.\frac{d}{d s}\right|_{0} \frac{1}{\Gamma(s)} \int_{0}^{\infty} d t t^{s-1} \operatorname{tr}\left\{-\frac{1}{4} \exp -t\left(\begin{array}{cc}
-D^{2} \delta_{i j} & -\epsilon_{i m n} F_{m n} \\
\epsilon_{m n j} F_{m n} & -D^{2}
\end{array}\right)+\exp -t\left(-D^{2}\right)\right\} .
$$

Employing the expansion of eq. (19) and computing functional traces in momentum space with [20]

$$
<p|f| q>=\frac{1}{(2 \pi)^{3 / 2}} f(p-q)
$$

we find that the contribution to the two point function coming from (20) is

$$
\begin{gathered}
W_{2}^{(1)}(A)=-\left.\frac{d}{d s}\right|_{0} \frac{1}{\Gamma(s)} \int_{0}^{\infty} d t t^{s-1} \int \frac{d p d q}{(2 \pi)^{3}}\left\{-\frac{1}{4}(-t)^{2} \int_{0}^{1} d u\right. \\
e^{-\left[(1-u) p^{2}+u q^{2}\right] t}\left[( - \epsilon _ { i m n } ) \left(f^{a p b} f_{m n}^{p}(p-q) \cos \frac{p \times q}{2}\right.\right. \\
\left.+d^{a p b} f_{m n}^{p}(p-q) \sin \frac{p \times q}{2}\right)\left(\epsilon_{r s i}\right)\left(f^{b q a} f_{r s}^{q}(q-p) \cos \frac{q \times p}{2}\right. \\
\left.\left.\left.+d^{b q a} f_{r s}^{q}(q-p) \sin \frac{q \times p}{2}\right)\right]\right\} \quad\left(f_{i j}^{a} \equiv \partial_{i} A_{j}^{a}-\partial_{j} A_{i}^{a}\right) .
\end{gathered}
$$

Upon making the usual shift in momentum variables, this becomes

$$
\begin{gathered}
=\left.\frac{N}{2} \frac{d}{d s}\right|_{0} \frac{1}{\Gamma(s)} \int_{0}^{\infty} d t t^{s+1} \int \frac{d p d q}{(2 \pi)^{3}} \int_{0}^{1} d u e^{-\left[q^{2}+u(1-u) p^{2}\right] t} \\
f_{m n}^{a}(p) f_{m n}^{b}(-p)\left(\delta^{a b}-\delta^{a 0} \delta^{b 0} \cos (p \times q)\right) .
\end{gathered}
$$

The standard integrals [21]

$$
\int \frac{d^{n} k}{(2 \pi)^{n}} e^{-k^{2} t}=\frac{1}{(4 \pi t)^{n / 2}}
$$




$$
\begin{gathered}
\int_{0}^{\infty} d t t^{\nu-1} e^{-A t}=\Gamma(\nu) A^{-\nu} \\
\int_{0}^{\infty} d t t^{\nu-1} e^{-\gamma t-\beta / t}=2\left(\frac{\beta}{\gamma}\right)^{\nu / 2} K_{ \pm \nu}(2 \sqrt{\beta \gamma})
\end{gathered}
$$

can be used to reduce (23) to

$$
\begin{array}{r}
W_{2}^{(1)}(A)=\left.\frac{N}{2} \frac{d}{d s}\right|_{0} \frac{1}{\Gamma(s)} \int \frac{d^{3} p}{(4 \pi)^{3 / 2}} \int_{0}^{1} d u f_{m n}^{a}(p) f_{m n}^{a}(-p) \\
{\left[\Gamma\left(s+\frac{1}{2}\right)\left(u(1-u) p^{2}\right)^{-s-1 / 2} \delta^{a b}\right.} \\
\left.\left.-2\left(\frac{\tilde{p}^{2}}{4 u(1-u) p^{2}}\right)^{\frac{s+1 / 2}{2}} K_{s+1 / 2}\left(2 \sqrt{\frac{u(1-u) p^{2} \tilde{p}^{2}}{4}}\right) \delta^{a 0} \delta^{b 0}\right)\right]
\end{array}
$$

where $p \times q \equiv \tilde{p} \cdot q$. It is now possible to evaluate $\left.\frac{d}{d s}\right|_{0}$ in $(25)$ explicitly, leaving us with

$$
\begin{aligned}
W_{2}^{(1)}(A) & =\frac{N}{2} \int \frac{d^{3} p}{(4 \pi)^{3 / 2}} \int_{0}^{1} d u f_{m n}^{a}(p) f_{m n}^{b}(-p)\left[\sqrt{\frac{\pi}{u(1-u) p^{2}}} \delta^{a b}\right. \\
- & \left.\left(\frac{4 \tilde{p}^{2}}{u(1-u) p^{2}}\right)^{1 / 4} K_{1 / 2}\left(\sqrt{u(1-u) p^{2} \tilde{p}^{2}}\right) \delta^{a 0} \delta^{b 0}\right] .
\end{aligned}
$$

Since $K_{ \pm 1 / 2}(z)=\sqrt{\frac{\pi}{2 z}} e^{-z}$, it is possible to compute both integrals over $u$ in (26) using [21]

$$
\begin{gathered}
\int_{0}^{1} d u u^{a-1}(1-u)^{b-1}=\frac{\Gamma(a) \Gamma(b)}{\Gamma(a+b)} \\
\int_{0}^{1} d u \frac{1}{\sqrt{u(1-u)}} e^{-A \sqrt{u(1-u)}}=2 \int_{0}^{\pi / 2} d \theta e^{-\frac{A}{2} \cos \theta}=\pi I_{0}(A / 2)
\end{gathered}
$$

leaving us with

$$
W_{2}^{(1)}(A)=\frac{N}{16} \int \frac{d^{3} p}{\sqrt{p^{2}}}\left[f_{m n}^{a}(p) f_{m n}^{b}(-p)\left(\delta^{a b}-I_{0}\left(\frac{\sqrt{p^{2} \tilde{p}^{2}}}{2}\right) \delta^{a 0} \delta^{b 0}\right)\right]
$$

In the limit $\theta^{\mu \nu} \rightarrow 0$ (so that $\left.\tilde{p}^{2} \rightarrow 0\right), I_{0}\left(\frac{\sqrt{p^{2} \tilde{p}^{2}}}{2}\right) \rightarrow 1$ leaving only the $S U(N)$ contribution to $(28)$. 
We can now consider the loss of phase associated with the replacement of eq. (12). This phase has been discussed extensively in $[1,10-12]$; it is associated with so-called $\eta$-function,

$$
\eta(s)=\frac{1}{\Gamma\left(\frac{s+1}{2}\right)} \int_{0}^{\infty} d t t^{\frac{s-1}{2}} \operatorname{Tr}\left(H_{I} e^{-H_{I}^{2} t}\right) .
$$

If a parameter $\lambda$ is inserted into $H_{I}$ so that $H_{I}(\lambda=1)=H_{I}$, then from (29) it is easily seen that

$$
\frac{d \eta_{\lambda}(s)}{d \lambda}=\frac{-s}{\Gamma\left(\frac{s+1}{2}\right)} \int_{0}^{\infty} d t t^{\frac{s-1}{2}} \operatorname{Tr}\left(\frac{d H_{I}(\lambda)}{d \lambda} e^{-H_{I}^{2}(\lambda) t}\right) .
$$

As only $\eta(0)$ is required to determine the phase we are interested in, it is sufficient to compute the poles arising in the integral over $t$ in (30) on account of the explicit factor of $s$ arising in front of the integral.

If now

$$
H_{I}(\lambda) \equiv\left(\begin{array}{cc}
\epsilon_{i p j}\left(\partial_{p}-i \lambda\left[A_{p}\right)\right. & -\left(\partial_{i}-i \lambda\left[A_{i}\right)\right. \\
\left(\partial_{j}-i \lambda\left[A_{j}\right)\right. & 0
\end{array}\right)
$$

then it is possible to expand the right side of (30) in powers of the background field using a second expansion due to Schwinger [20]

$$
\begin{gathered}
e^{-\left(H_{0}+H_{1}\right) t}=\left[e^{-H_{0} t}+(-t) \int_{0}^{1} d u e^{-(1-u) H_{0} t} H_{1} e^{-u H_{0} t}\right. \\
+(-t)^{2} \int_{0}^{1} d u u \int_{0}^{1} d v e^{-(1-u) H_{0} t} H_{1} e^{-u(1-v) H_{0} t} \\
\left.H_{1} e^{-u v H_{0} t}+\ldots\right] .
\end{gathered}
$$

From (15), (31) and (32), it is evident that the contribution to (30) that is bilinear in the background field $A_{\mu}$ is (upon following the approach used above with the $\zeta$-function)

$$
\frac{d \eta_{\lambda}^{(2)}(s)}{d \lambda}=\frac{N \lambda s}{\Gamma\left(\frac{s+1}{2}\right)} \int_{0}^{\infty} d t t^{\frac{s+1}{2}} \int_{0}^{1} d u \int \frac{d p d q}{(2 \pi)^{3}} e^{-\left(q^{2}+u(1-u) p^{2}\right) t}
$$




$$
\left(\epsilon_{i j k} A_{i}^{a}(p) f_{j k}^{b}(-p)\right)\left(\delta^{a b}-\delta^{a 0} \delta^{b 0} \cos (p \times q)\right) .
$$

The integrals appearing in (33) are identical in form to these in (23); just as we obtain (28) we find that

$$
\begin{array}{r}
\frac{d \eta_{\lambda}^{(2)}(s)}{d \lambda}=\frac{N \lambda s}{\Gamma\left(\frac{s+1}{2}\right)} \int_{0}^{1} d u \int \frac{d p}{(4 \pi)^{3 / 2}} \epsilon_{i j k} A_{i}^{a}(p) f_{j k}^{b}(-p) \\
{\left[\delta^{a b} \Gamma\left(\frac{s}{2}\right)\left(u(1-u) p^{2}\right)^{-s / 2}\right.} \\
\left.-2 \delta^{a 0} \delta^{b 0}\left(\frac{\tilde{p}^{2}}{4 u(1-u) p^{2}}\right)^{s / 4} K_{s / 2}\left(\sqrt{u(1-u) p^{2} \tilde{p}^{2}}\right)\right] .
\end{array}
$$

In the limit $x \rightarrow 0, K_{\nu}(x) \rightarrow 2^{\nu-1} \Gamma(\nu) x^{-\nu}$ and thus when $\theta_{\mu \nu} \rightarrow 0\left(\tilde{p}^{2} \rightarrow 0\right)$, (34) reduces to

$$
\begin{gathered}
\approx \frac{N \lambda s}{\Gamma\left(\frac{s+1}{2}\right)} \int_{0}^{1} d u \int \frac{d p}{(4 \pi)^{3 / 2}} \epsilon_{i j k} A_{i}^{a}(p) f_{j k}^{b}(-p) \\
{\left[\Gamma\left(\frac{s}{2}\right)\left(u(1-u) p^{2}\right)^{-s / 2}\right]} \\
{\left[\delta^{a b}-\delta^{a 0} \delta^{b 0}\right] .}
\end{gathered}
$$

As in the case of the $\zeta$-function, only the $S U(N)$ contribution to $\frac{d}{d \lambda} \eta_{\lambda}(0)$ survives in the commutative limit.

\section{Discussion}

Chern Simons theory is difficult to regulate on account of the presence of the tensor $\epsilon_{i j k}$. Operator regularization appears however to be a suitable way of dealing with this problem as it does not involve altering the original Lagrangian. This permits one to deal with both the usual commutative $S U(N)$ Chern Simons model and as well noncommutative $U(N)$ Chern Simons theory. An analysis of the one loop two point function done here has shown 
that in the commutative limit, the non-commutative $U(N)$ model smoothly reduces to the commutative $S U(N)$ model.

\section{Acknowledgements}

Must of this work was done while the author was a guest at the University of Sao Paulo; he is particularly grateful to F. Brandt and J. Frenkel for their hospitality there. R. and D. MacKenzie had useful suggestions. 


\section{References}

[1] E. Witten, Comm. Math. Phys. 121, 351 (1989).

[2] D. Birmingham, M. Blau, M. Rakowski and G. Thompson, Phys. Rep. 209, 129 (1991).

[3] SenHu, "Chern-Simons-Witten Theory" (World Scientific, Singapore, 2002).

[4] R. Szabo, hep-th 0109162 .

[5] M. Douglas and N.A. Nekrasov, hep-th 0106048.

[6] G.H. Chen and Y.S. Wu, Nucl. Phys. B593, 562 (2001)

N. Grandi and G.A. Silva, Phys. Lett. B507, 345 (2001)

M.M. Sheikh-Jabbari, Phys. Lett. B510, 247 (2001)

A. Das and M.M. Sheikh-Jabbari, JHEP 0106, 28 (2001)

C.P. Martin, Phys. Lett. B515, 185 (2001).

[7] A.S. Bichl, J.M. Grimstrup, V. Putz and M. Schweda, JHEP 0007, 046 (2001).

[8] D.G.C. McKeon and T.N. Sherry, Phys. Rev. Lett. 59, 532 (1987), Phys. Rev. D35, 854 (1987)

[9] A. Salam and J. Strathdee, Nucl. Phys. B90, 203 (1975)

J. Dawker and R. Critchley, Phys. Rev. D13, 3224 (1976)

S. Hawking, Comm. Math. Phys. 55, 133 (1977). 
[10] P. Gilkey, "Invariance Theory, the Heat Equation, and the Atiyah-Singer Index Theorem", Publish or Perish Inc. (1984).

[11] D. Birmingham, R. Kantowski and M. Rakowski, Phys. Lett. B 151, 121 (1990); Phys. Rev. D4, 3476 (1990).

[12] D.G.C. McKeon and T.N. Sherry, Ann. of Phys. 218, 325 (1992).

[13] D.G.C. McKeon, Can. J. Phys. 68, 1291 (1990).

[14] D.G.C. McKeon and C. Wong, Int. J. of Mod. Phys. A10, 2181 (1995).

[15] F.A. Dilkes, L.C. Martin, D.G.C. McKeon and T.N. Sherry, Int. J. of Mod. Phys. A14, 463 (1999).

[16] B.S. DeWitt, Phys. Rev. 162, 1195, 1239 (1967).

[17] G. 't Hooft, Nucl. Phys. B61, 455 (1973).

[18] L. Abbott, Nucl. Phys. B185, 189 (1981).

[19] L. Bonara and M. Salizzoni, Phys. Lett. B504, 80 (2001).

[20] J. Schwinger, Phys. Rev. 82, 664 (1951).

[21] I. Gradshteyn and M. Ryzhik, Table of Integrals, Series and Products (Academic Press, New York 1980). 